\title{
Estratégias de ensino das terapias alternativas e complementares na graduação em Enfermagem: revisão integrativa
}

\author{
Strategies to teach alternative and complementary therapies in nursing: an integrative review
}

Estrategias de enseñanza de terapias alternativas y complementarias en curso de graduación en Enfermería: revisión integrativa

Natália Chantal Magalhães da Silva ${ }^{1}$, Denise Hollanda lunes² , Zélia Marilda Rodrigues Resck² Mirelle Inácio Soares ${ }^{4}$, Deusdete Inácio de Souza Junior ${ }^{5}$, Neireana Florencio Vieira ${ }^{6}$

\footnotetext{
${ }^{1}$ Enfermeira. Discente do Programa de Pós-Graduação em Enfermagem (PPGENF), nível Mestrado, da Universidade Federal de Alfenas (UNIFAL-MG). Alfenas, MG, Brasil. E-mail: naty chantal@hotmail.com.

${ }^{2}$ Fisioterapeuta, Doutora em Ciências Médicas. Professora Adjunta III da UNIFAL-MG. Alfenas, MG, Brasil. E-mail: deniseiunes@unifal-mg.edu.br.

${ }^{3}$ Enfermeira, Doutora em Enfermagem. Professora Adjunta IV da UNIFAL-MG. Alfenas, MG, Brasil. E-mail: zeliar@unifal-mg.edu.br.

${ }^{4}$ Enfermeira. Discente do PPGENF/UNIFAL-MG, nível Mestrado. Alfenas, MG, Brasil. E-mail: mirelle soares83@yahoo.com.br.

${ }^{5}$ Enfermeiro. Discente do PPGENF/UNIFAL-MG, nível Mestrado. Alfenas, MG, Brasil. E-mail: unijunior6@yahoo.com.br.

${ }^{6}$ Enfermeira. Discente do PPGENF/UNIFAL-MG, nível Mestrado. Alfenas, MG, Brasil. E-mail: naninha enf@yahoo.com.br.
}

\section{RESUMO}

A ausência de discussões que forneçam esclarecimentos sobre o ensino das terapias alternativas e complementares durante a graduação em Enfermagem reflete a necessidade de desenvolvimento de pesquisas que abordem a temática. Este estudo objetivou identificar, na literatura científica, estratégias de ensino das terapias alternativas e complementares na graduação em Enfermagem. Utilizou-se da revisão integrativa de literatura como metodologia norteadora. O levantamento bibliográfico foi realizado nas bases de dados SCIENCE DIRECT, LILACS e MEDLINE. De acordo com os artigos que compuseram este estudo, as propostas de ensino para a inserção dessas terapias na área acadêmica são: palestras, cursos teóricos e grupos de discussão; disciplina optativa; inserção de disciplina no currículo e incentivo à pesquisa. Entretanto, são necessários estudos que comprovem a eficácia dessas estratégias para que seja possível inserir no Projeto Político Pedagógico do curso de graduação em Enfermagem esses métodos terapêuticos, possibilitando assim a consolidação de tais práticas.

Descritores: Educação em Enfermagem; Educação Superior; Terapias Complementares.

\section{ASBTRACT}

The lack of discussions that clarify the teaching of alternative and complementary therapies in undergraduate nursing programs shows the need for developing research on this topic. The objective of this study was to identify, in scientific literature, the strategies for teaching alternative and complementary therapies in nursing undergraduate programs. The guiding methodology used was integrative review. The search was performed on SCIENCE DIRECT, LILACS and MEDLINE databases. According to the articles included in this study, the proposals for including these therapies in the program are by: lectures, theoretical courses and discussion groups. However, studies should be conducted to confirm the efficacy of these strategies so these therapeutic methods can be included in the Pedagogical Political Project of the nursing undergraduate program, thus allowing for the consolidation of those practices.

Descriptors: Education, Nursing; Education, Higher; Complementary Therapies.

\section{RESUMEN}

La falta de discusiones aclaratorias sobre enseñanza de terapias alternativas y complementarias durante el curso de graduación en Enfermería, refleja la necesidad de desarrollar investigaciones que aborden la temática. Se objetivó identificar en la literatura científica, estrategias de enseñanza de terapias alternativas y complementarias en graduación en Enfermería. Se aplicó revisión integrativa de literatura como metodología orientadora. El relevamiento bibliográfico fue realizado en las bases SCIENCE DIRECT, LILACS y MEDLINE. De acuerdo con los artículos que integraron este estudio, las propuestas de enseñanza para insertar tales terapias en el área académica son: talleres, cursos teóricos y grupos de discusión; disciplina optativa; inserción de la disciplina en el programa e incentivación de la investigación. No obstante, son necesarios estudios que comprueben la eficacia de tales estrategias para posibilitar la insertación en el Proyecto Político Pedagógico del curso de graduación en Enfermería dichos métodos terapéuticos, apuntando a la consolidación de tales prácticas.

Descriptores: Educación en Enfermería; Educación Superior; Terapias Complementarias. 


\section{INTRODUÇÃO}

O aumento da aceitação e confiabilidade das terapias alternativas e complementares, bem como o despreparo dos profissionais de saúde em incluir essas práticas no cuidado fornecido durante o atendimento nos serviços, vem possibilitando reflexões acerca do ensino acadêmico das mesmas(1). Também denominadas como não convencionais, essas terapias caracterizam-se como técnicas ou métodos que objetivam a assistência de saúde ao indivíduo, seja na prevenção, tratamento ou recuperação. Diferentemente da terapia convencional, cujo enfoque é a cura pela intervenção direta no órgão ou parte adoecida, nessas terapias o foco é o ser humano como um todo(2-3).

Sob a perspectiva biomédica, o corpo humano é fragmentado e deve receber assistência de maneira específica e dissociada. Esse paradigma leva os profissionais de saúde a negligenciarem aspectos psicossociais, emocionais, espirituais, culturais e ambientais do indivíduo, afastando-se do cuidado holístico e integral, tão defendido na atualidade. Assim, a adoção das terapias alternativas e complementares na assistência à saúde em diferentes níveis, além de aumentar a disponibilidade de possíveis tratamentos e favorecer o alcance de melhores resultados no processo saúde-doença, contribui para o equilíbrio entre todas as dimensões do ser humano, atuando não só na parte física, mas também nas energias mais sutis que formam seu corpo $^{(1-4)}$.

O enfermeiro, por estar em contato direto com a população, tendo a oportunidade de educá-la e esclarecêla quanto ao uso, benéfico ou não, dessas técnicas, desempenha papel essencial nesse contexto(2). Entretanto, no Brasil, o desenvolvimento de estudos sobre o uso dessas terapias por profissionais da Enfermagem é ainda incipiente. Embora a pesquisa seja de pouca abrangência, a discussão sobre as práticas não convencionais vem avançando, obtendo regulamentação do exercício profissional por meio da Resolução COFEN 197/97, que reconhece esses métodos terapêuticos como especialidade e/ou qualificação da Enfermagem ${ }^{(5)}$.

Portanto, nos debates atuais assume-se como fundamental que os profissionais de Enfermagem sejam formados tendo acesso ao núcleo básico das terapias alternativas e complementares, contudo, não é o que se observa na prática acadêmica. Em uma pesquisa realizada em cinco escolas de Enfermagem do estado de São Paulo, a formação de enfermeiros com relação a tais técnicas ocorreu ao longo da carreira, em cursos de diferentes formatos, com caráter de educação continuada e especialização, não havendo um momento na graduação com a abordagem dessa temática ${ }^{(5)}$.

O fato das práticas alternativas e complementares estarem à mercê do currículo tradicional das Escolas de Enfermagem configura-se um círculo vicioso: os acadêmicos não recebem formação dessas terapias pelo fato dos docentes não deterem conhecimento amplo do assunto, consequentemente, formam profissionais incapazes de ensinar e praticar modelos não convencionais terapêuticos(3). São apontadas várias razões para a inclusão do ensino de métodos alternativos e complementares no planejamento das experiências do estudante e desenvolvimento da sensibilidade cultural do aluno, com informações que possam modelar as atitudes e o comportamento relatado na saúde e na doença, visto que, durante o período de graduação é que se adquire os conhecimentos básicos necessários sobre o processo saúde-doença e se desenvolve o interesse pela futura área de atuação(4).

A ausência de discussões e a escassez de estudos que forneçam esclarecimentos sobre a metodologia de ensino das terapias alternativas e complementares durante a graduação em Enfermagem, refletem a necessidade do desenvolvimento de pesquisas que abordem a temática. Os achados fornecidos pelas pesquisas científicas acerca da abordagem das terapias não convencionais no ensino superior permitirão a discussão sobre a melhor maneira de inserção desses métodos no currículo acadêmico, contribuindo, assim, para a melhoria da formação do enfermeiro.

Sendo assim, o objetivo deste estudo foi identificar, na literatura científica, estratégias de ensino das terapias alternativas e complementares na graduação em Enfermagem.

\section{MÉTODOS}

Utilizou-se da revisão integrativa de literatura como metodologia norteadora deste estudo. Esse método se configura como uma análise rigorosa de todas as pesquisas relacionadas a uma temática, seja um estudo teórico ou empírico ${ }^{(6-7)}$. Tem como objetivo, gerar uma 
compreensão de conceitos, teorias ou problemas de saúde relevantes para a Enfermagem ${ }^{(8-9)}$.

Este estudo cumpriu criteriosamente seis etapas. Visando alcançar o objetivo da revisão, a primeira fase consistiu na seleção da temática e escolha da questão norteadora: "Quais as estratégias de ensino das terapias alternativas e complementares na graduação em Enfermagem?".

O levantamento bibliográfico, segunda etapa, foi realizado nas bases de dados SCIENCE DIRECT, LILACS E MEDLINE. A busca ocorreu durante o mês de agosto de 2012 e teve como critérios de inclusão artigos publicados nos 10 últimos anos, na língua inglesa, espanhola ou portuguesa e disponíveis online em texto completo. Como estratégias de investigação, foram utilizadas: "therapies alternative" [Descritor de assunto] or "complementary therapies" [Descritor de assunto] and "nursing education" [Descritor de assunto]. Esse levantamento reuniu 91 citações ao término do processo de levantamento bibliográfico.

Na terceira etapa, com o objetivo de verificar se os estudos abordavam a temática estudada, foi realizada uma análise seletiva por meio da leitura dos resumos. Nessa fase, foram excluídos 73 artigos que não abordavam as terapias alternativas e complementares na graduação em Enfermagem, restando 18 para compor a revisão.

Na análise crítica, quarta fase, os 18 artigos foram lidos na íntegra e verificou-se que apenas seis apontavam estratégias de ensino das terapias alternativas e complementares, respondendo, assim, a questão norteadora proposta. A Figura 1 representa o número de artigos referente a cada base de dados que compôs a revisão integrativa.

Figura 1: Diagrama de coleta de dados.

\begin{tabular}{ccc} 
& Levantamento bibliográfico & \\
\hline SCIENCE DIRECT & LILACS & MEDLINE \\
35 & 5 & 51 \\
& & \\
& Análise seletiva & \\
SCIENCE DIRECT & LILACS & MEDLINE \\
10 & 2 & 6 \\
& & \\
& & MEDLINE \\
SCIENCE DIRECT & LILACS & 3 \\
1 & 2 & \\
\end{tabular}

Informações importantes foram extraídas dos estudos para posterior agrupamento e categorização dos resultados encontrados, caracterizando a quinta etapa da revisão.

A sexta e última etapa configura-se na elaboração da revisão final, contendo a discussão de informações pertinentes e detalhadas, sem omissão das evidências encontradas.

\section{RESULTADOS}

Na composição deste estudo, as pesquisas relacionadas às terapias alternativas e complementares na formação em Enfermagem ocorreram em três países, sendo três dos seis artigos provenientes dos Estados Unidos, dois advindos do Brasil e apenas um oriundo da
Austrália. Quatro artigos foram publicados na língua inglesa e dois na língua portuguesa. O Quadro 1 apresenta a caracterização dos estudos. 
Quadro 1: Síntese dos artigos que compuseram a revisão integrativa de acordo com os critérios de inclusão pré-estabelecidos e abordagem da temática norteadora, Alfenas, MG, Brasil, 2012.

\begin{tabular}{|c|c|c|c|c|}
\hline Autores & Considerações Temáticas & Metodologia & $\begin{array}{c}\text { Conceito de terapias } \\
\text { alternativas e } \\
\text { complementares }\end{array}$ & \begin{tabular}{|} 
Estratégias de ensino \\
das terapias \\
alternativas e \\
complementares na \\
graduação em \\
Enfermagem
\end{tabular} \\
\hline $\begin{array}{c}\text { BARROS, N. F.; TOVEY, } \\
\text { P. }\end{array}$ & $\begin{array}{l}\text { Explora o ensino das } \\
\text { terapias alternativas e } \\
\text { complementares em cinco } \\
\text { escolas de Enfermagem do } \\
\text { estado de São Paulo }\end{array}$ & Qualitativa & $\begin{array}{l}\text { Medidas que não fazem } \\
\text { parte das práticas } \\
\text { convencionais do modelo } \\
\text { biomédico, usadas para } \\
\text { promover saúde e bem- } \\
\text { estar, prevenir e tratar } \\
\text { doenças. }\end{array}$ & $\begin{array}{c}\text { Seminários, cursos } \\
\text { teóricos e palestras; } \\
\text { incentivo às pesquisas }\end{array}$ \\
\hline $\begin{array}{l}\text { TROVO, M. M.; SILVA, } \\
\text { M. J. P.; LEÃO, E. R. }\end{array}$ & $\begin{array}{l}\text { Analisa o conhecimento de } \\
\text { alunos da graduação em } \\
\text { Enfermagem em relação às } \\
\text { terapias alternativas/ } \\
\text { complementares }\end{array}$ & $\begin{array}{l}\text { Quantitativa, } \\
\text { exploratória, } \\
\text { descritiva, } \\
\text { transversal }\end{array}$ & $\begin{array}{c}\text { Técnicas que visam à } \\
\text { assistência à saúde do } \\
\text { indivíduo, seja na } \\
\text { prevenção, tratamento } \\
\text { ou cura, considerando-o } \\
\text { como mente, corpo e } \\
\text { espírito. } \\
\end{array}$ & Disciplina optativa \\
\hline $\begin{array}{l}\text { BOOTH-LAFORCE, C.; } \\
\text { SCOTT, C. S.; } \\
\text { KEITKEMPER, M.; } \\
\text { CORNMAN, J.; LAN, M.; } \\
\text { BOND, E. F.; } \\
\text { SWANSON, K. M. }\end{array}$ & $\begin{array}{l}\text { Avalia o impacto do } \\
\text { programa de } \\
\text { desenvolvimento do corpo } \\
\text { docente sobre as atitudes e } \\
\text { competências da medicina } \\
\text { alternativa e complementar }\end{array}$ & Quantitativa & & Disciplina optativa \\
\hline $\begin{array}{l}\text { LEE, M. Y.; BENN, R.; } \\
\text { WIMSATT, L.; } \\
\text { COMMAN, J.; } \\
\text { HEDGECOCK, J.; } \\
\text { GERIK, S.; ZELLER, J.; } \\
\text { KREITZER, M. J.; } \\
\text { ALLWEISS, P.; } \\
\text { FINKLESTEIN, C.; } \\
\text { HARAMATI, A. }\end{array}$ & $\begin{array}{c}\text { Aborda estratégias } \\
\text { organizacionais e de } \\
\text { instrução para integrar a } \\
\text { medicina alternativa e } \\
\text { complementar no currículo, } \\
\text { destacando as atividades } \\
\text { relacionadas com o } \\
\text { desenvolvimento do corpo } \\
\text { docente }\end{array}$ & Qualitativa & & $\begin{array}{l}\text { Grupos de discussão; } \\
\text { disciplina optativa }\end{array}$ \\
\hline $\begin{array}{c}\text { GASTER, B.; } \\
\text { UNTERBORN, J. N.; } \\
\text { SCOTT, R. B.; } \\
\text { SCHNEEWEISS, R. }\end{array}$ & $\begin{array}{l}\text { Traça princípios } \\
\text { fundamentais que norteiam } \\
\text { os programas de educação } \\
\text { complementar e medicina } \\
\text { alternativa no } \\
\text { desenvolvimento de cursos } \\
\text { de saúde }\end{array}$ & Qualitativa & $\begin{array}{c}\text { Práticas de cura, } \\
\text { tecnologias, produtos e } \\
\text { perspectivas que não são } \\
\text { componentes } \\
\text { estabelecidos pela } \\
\text { medicina convencional. }\end{array}$ & $\begin{array}{c}\text { Disciplina optativa; } \\
\text { incentivo às pesquisas }\end{array}$ \\
\hline $\begin{array}{l}\text { RIET, P. V. D.; FRANCIS, } \\
\text { L.; LEVETT-JONES, T. }\end{array}$ & $\begin{array}{c}\text { Descreve as razões para a } \\
\text { introdução do curso eletivo } \\
\text { de terapias } \\
\text { complementares na } \\
\text { Universidade de Newcastle }\end{array}$ & Qualitativa & & $\begin{array}{l}\text { Inserção de disciplina } \\
\text { no currículo }\end{array}$ \\
\hline
\end{tabular}

O conceito das práticas alternativas e complementares foi abordado em $50 \%$ dos estudos. Essas terapias são caracterizadas como "medidas que não fazem parte das práticas convencionais do modelo biomédico, usadas para promover saúde e bem-estar, prevenir e tratar doenças"(5). De maneira semelhante, podem ser qualificadas como "técnicas que visam à assistência à saúde do indivíduo, seja na prevenção, tratamento ou cura, considerando-o como mente, corpo e espírito e não como um conjunto de partes isoladas"(10). As técnicas terapêuticas não convencionais: "são muitas vezes referidas como medicina complementar e alternativa, definidas como práticas de cura, tecnologias, produtos e perspectivas que não são componentes 
estabelecidos pela medicina convencional. Essas terapias são derivadas de: tradições de cura, incluindo aromaterapia e reflexologia; uso do toque terapêutico; terapias físicas, incluindo massagem e terapias energéticas, como por exemplo, a meditação"(11).

A abordagem das terapias alternativas e complementares na Enfermagem foi mencionada como sendo essencial durante a formação na graduação. Assim sendo, foram construídas categorias de acordo com as estratégias de ensino, já utilizadas por algumas Instituições ou sugeridas por pesquisadores, para a inserção dessas terapias na área acadêmica.

\section{Palestras, cursos teóricos e grupos de discussão}

Os cursos teóricos e palestras são a maneira mais eficiente e rápida para atingir grandes grupos de alunos ${ }^{(5)}$. Também se faz importante a instituição de grupos de discussão de estudantes em torno das práticas holísticas, para aprofundamento do tema e fornecimento de informações pertinentes para a prática clínica ${ }^{(12)}$.

Atividades que incentivem os discentes a discutir sobre as terapias, bem como seus benefícios, malefícios, suas indicações e contraindicações, estimulam o aprendizado de novas formas de tratamento e prevenção de doenças e agravos. É necessário que o profissional de Enfermagem esteja preparado para esclarecer dúvidas e fornecer alternativas terapêuticas para os indivíduos que frequentam os serviços de saúde ${ }^{(1)}$.

\section{Disciplina optativa}

Disciplinas eletivas facilitam a inovação e diferenciação curricular, contornando alguns obstáculos institucionais, testando novos materiais ou estratégias de ensino e descobrindo interesses em determinados conteúdos. No entanto, mesmo que para um especifico número de alunos, é necessário um quadro de docentes que detenham conhecimento amplo e claro sobre os métodos alternativos e complementares e, infelizmente, são raros os educadores com essas características, apesar do uso difundido das terapias ${ }^{(12-13)}$.

\section{Inserção de disciplina no currículo}

A inserção de disciplinas voltadas para conteúdos sobre métodos terapêuticos não convencionais permite incorporar o uso dessas terapias na assistência prestada em campos de estágio e avança na busca por assegurar a integralidade da atenção e a qualidade e humanização no atendimento. A universidade tem um papel relevante na formação dos profissionais para atuarem com competência nos serviços de saúde pública e privada, cabendo à instituição conceder sustentação para o desenvolvimento de uma formação generalista que contemple o cuidado holístico(2).

\section{Incentivo às pesquisas}

O ensino das práticas não convencionais e a utilização das mesmas em pacientes apontando para o que funciona e o que é prejudicial, tornam-se possíveis por meio do desenvolvimento de pesquisas para a comprovação científica das mesmas. Os conhecimentos produzidos permitirão aos alunos ampliar a visão sobre o possível atendimento com terapias não convencionais, além de instrumentalizá-los para responder às perguntas que surgirem. Estudos possibilitam avaliar quais métodos terapêuticos são mais indicados para determinada condição médica, já que algumas condições são mais adequadamente tratadas com práticas alternativas e complementares que outras ${ }^{(14)}$.

\section{DISCUSSÃO}

O ensino da graduação em Enfermagem se defronta com profundas mudanças na formação profissional diante do desafio de inovar, reformar e transformar métodos acadêmicos. A ausência da abordagem que enfatiza a assistência baseada nas terapias holísticas no nível superior, leva a questionar a crescente valorização das práticas alternativas e complementares no mundo(11).

É relevante a necessidade de se incluir nos cursos de graduação em Enfermagem informações sobre o uso das terapias alternativas e complementares nos serviços de atenção primária, secundária e terciária, para que essas se façam presentes na integralidade do cuidado fornecido. Os enfermeiros na práxis e futuros profissionais devem compreender a legislação que assegura o desenvolvimento de técnicas não convencionais, com a devida valorização dessas práticas(2). É preciso que os acadêmicos tenham acesso à formas alternativas de assistência, de modo que o curso proporcione aos estudantes meios necessários para organizar, prever, resolver problemas, sintetizar e refletir sobre a sua aprendizagem para permitir o desenvolvimento de um 
conhecimento profissional teórico e prático de terapias complementares ${ }^{(11)}$.

Discute-se muito sobre os diferentes tipos de estratégias para o ensino dessas práticas no ensino superior. Essas metodologias de ensino devem permitir a abordagem das diferenças filosóficas e culturais que dão suporte a cada terapia, com temas que introduzam os alunos em terapêuticas específicas e que possibilitem a reflexão sobre a necessidade de incluir esses métodos não convencionais na prática da Enfermagem ${ }^{(5)}$.

Dessa forma, assume-se como premissa fundamental que, tanto enfermeiros quanto os demais profissionais da saúde, sejam formados tendo acesso ao conteúdo dessas terapias e a resultados de pesquisas sobre o uso desses métodos. Рara que isso se torne possível, é necessário o estímulo ao desenvolvimento de estudos que avaliem o conhecimento e percepções de enfermeiros, graduandos e docentes acerca do tema, bem como estudos que possibilitem comprovar ou negar a eficácia de determinada terapia ${ }^{(5)}$.

\section{CONCLUSÃO}

A discussão sobre as estratégias de ensino das terapias alternativas e complementares na graduação em Enfermagem, sob a perspectiva de diferentes autores, possibilitou a identificação de metodologias que podem ser utilizadas no processo ensino-aprendizagem dessas

\section{REFERÊNCIAS}

1. Barbosa MA, Siqueira KM, Brasil VV, Bezerra ALQ. Crenças populares e recursos alternativos como práticas de saúde. Rev. Enferm. UERJ [Internet]. 2004 [acesso em: $20 \mathrm{dez}$

2013];12(1):38-43. Disponível em:

http://www.facenf.ueri.br/v12n1/v12n1a06.pdf.

2. Gavin ROS, Oliveira MHP, Gherardi-Donato ECS. Terapias alternativas complementares: uma visão do conhecimento dos acadêmicos de enfermagem. Ciênc. cuid. saúde [Internet]. 2010 [acesso em: 20 dez 2013];12(4):760-5. Disponível em: http://periodicos.uem.br/ojs/index.php/CiencCuidSaude/article/ view/13827.

3. Seravalle L, Boog MCF. Introduçäo à discussäo sobre o ensino de práticas alternativas em saúde. Saúde em Debate.

1996;51(1):82-8.

4. Trovó MM, Silva MJP. Terapias alternativas / complementares a visão do graduando de enfermagem. Rev Esc Enferm USP [Internet]. 2002 [acesso em: 20 dez 2013];36(1):75-9. Disponível em: http://dx.doi.org/10.1590/S0080-62342002000100012.

5. Barros NF, Tovey P. O ensino das terapias alternativas e complementares em escolas de enfermagem. Rev Gaucha Enferm [Internet]. 2007 [acesso em: $20 \mathrm{dez}$ 2013];28(2):207-14. Disponivel em:

http://seer.ufrgs.br/RevistaGauchadeEnfermagem/article/view/ $\underline{3165}$. práticas. Tanto a realização de palestras, cursos teóricos e grupos de discussão, como a inserção de disciplina opcional ou obrigatória no currículo, bem como o incentivo à pesquisa científica favorecem a abordagem, nos mais diferentes aspectos, do conteúdo que envolve essas terapias.

São poucos os estudos que tratam dos métodos de ensino de tais práticas na educação de nível superior da Enfermagem. A escassez de informações concisas e específicas sobre essa temática limita a visão holística e de integralidade requerida ao enfermeiro, o que gera uma deficiência na sua formação básica, trazendo prejuízo no seu desempenho profissional.

Além disso, as atitudes dos estudantes do presente se refletem no profissional do futuro e tendências no campo das terapias não convencionais podem trazer implicações para o relacionamento enfermeiro-paciente e para o futuro do cuidado interdisciplinar. Portanto, cabe a universidade a responsabilidade de inserir no Projeto Político Pedagógico do curso de graduação em Enfermagem a melhor estratégia de ensino desses métodos terapêuticos, possibilitando assim a estabilização e consolidação dessas práticas. Entretanto, é necessária a realização de estudos que comprovem a eficácia das estratégias de ensino das terapias alternativas e complementares, identificadas nesta pesquisa, na graduação em Enfermagem.

6. Whittemore R, Knafl K. The integrative review: updated methodology. J Adv Nurs [Internet]. 2005 [acesso em: $20 \mathrm{dez}$ 2013];52(5):546-53. Disponível em:

http://dx.doi.org/10.1111/j.1365-2648.2005.03621.x.

7. Mendes KDS, Silveira RCCP, Galvão CM. Revisão integrativa: método de pesquisa para a incorporação de evidências na saúde e na enfermagem. Texto Contexto Enferm [Internet]. 2008 [acesso em: $20 \mathrm{dez} 2013$ ];17(4):758-64. Disponível em: http://dx.doi.org/10.1590/S0104-07072008000400018. 8. Souza MT, Silva MD, Carvalho R. Revisão integrativa: o que é e como fazer. Einstein [Internet]. 2010 [acesso em: $20 \mathrm{dez}$ 2013];8(1):102-6. Disponível em:

http://apps.einstein.br/revista/arquivos/PDF/1134-

Einsteinv8n1_p102-106 port.pdf.

9. Polit DF, Beck CT, Hungler BP. Fundamentos de Pesquisa em Enfermagem: métodos, avaliação e utilização. $5^{\text {a }}$ ed. Porto Alegre: Artes Médicas; 2004

10. Trovo MM, Silva MJP, Leão ER. Terapias alternativas/complementares no ensino público e privado: análise do conhecimento dos acadêmicos de enfermagem. Rev Lat Am Enfermagem [Internet]. 2003 [acesso em: $20 \mathrm{dez}$ 2013];11(4): 483-9. Disponível em:

http://dx.doi.org/10.1590/S0104-11692003000400011.

11. van der Riet $P$, Francis $L$, Levett-Jones T. Complementary therapies in healthcare: design, implementation and evaluation 
of an elective course for undergraduate students. Nurse Educ Pract [Internet]. 2011 [acesso em: 20 dez 2013];11(2):146-52. Disponivel em: http://dx.doi.org/10.1016/j.nepr.2010.10.002. 12. Lee MY, Benn R, Wimsatt L, Cornman J, Hedgecock J, Gerik $\mathrm{S}$, et al. Integrating complementary and alternative medicine instruction into health professions education: organizational and instructional strategies. Acad Med [Internet]. 2007 [acesso em: 20 dez 2013];82(10):939-45. Disponível em: http://dx.doi.org/10.1097/ACM.0b013e318149ebf8. 13. Booth-Laforce C, Scott CS, Heitkemper MM, Cornman BJ, Lan MC, Bond EF, et al. Complementary and Alternative Medicine (CAM) attitudes and competencies of nursing students and faculty: results of integrating CAM into the nursing curriculum. J Prof Nurs [Internet]. 2010 [acesso em: $20 \mathrm{dez}$ 2013];26(5):293-300. Disponível em:

http://dx.doi.org/10.1016/j.profnurs.2010.03.003.

14. Gaster B, Unterborn JN, Scott RB, Schneeweiss R. What should students learn about complementary and alternative medicine? Acad Med [Internet]. 2007 [acesso em: 20 dez 2013];82(10):934-8. Disponível em: http://dx.doi.org/10.1097/ACM.0b013e318149eb56.

Artigo recebido em 08/10/2012.

Aprovado para publicação em 11/06/2013.

Artigo publicado em 31/12/2013. 